\title{
THE LYING ORACLE GAME WITH A BIASED COIN
}

\author{
ROBB KOETHER * AND \\ MARCUS PENDERGRASS, ${ }^{* *}$ Hampden-Sydney College \\ JOHN OSOINACH, ${ }^{* * *}$ Millsaps College
}

\begin{abstract}
The lying oracle problem is a problem of finding the optimal strategies in a two-person game where an oracle predicts the outcomes of coin flips and a player bets on the outcomes. The oracle announces whether the coin will land heads or tails, but may at times lie. We analyze the variant of the game which uses a biased coin, where the probability $p$ that the coin lands heads is common knowledge. We determine optimal strategies for both the oracle and player, and we give an explicit expression for the expected payoff to the player when the coin is flipped $n$ times and the oracle may lie at most $k$ times.
\end{abstract}

Keywords: Lying oracle; probabilistic game; optimal strategy; expected payoff; harmonic mean

2000 Mathematics Subject Classification: Primary 91A60

Secondary 91A05; 60C05; 00A08

\section{Introduction}

The lying oracle problem as given in [3] is the problem of finding the optimal strategies in a two-person game between an oracle and a bettor (the player). The oracle agrees to flip a coin $n$ times and will predict the outcome accurately, except that the oracle may lie up to $k$ times. Before each prediction, the player places a bet of any amount up to the player's current holdings. The oracle will then announce the outcome, after which the player must state the outcome on which to bet. The problem is to determine the optimal wagers and strategy for the player, and to determine with what probabilities the oracle should tell the truth and lie about the coin's outcome.

It was shown in [3] that in the game of $n$ flips and $k$ lies, there is a unique critical wager $w_{\mathrm{c}}=w_{\mathrm{c}}(n, k)$ (represented as a proportion of the player's current holdings) such that any wager $w$ satisfying $w_{\mathrm{c}} \leq w \leq 1$ is optimal. Furthermore, given that $w_{\mathrm{c}} \leq w \leq 1$, the oracle should tell the truth with probability $P_{t}=\frac{1}{2}+\frac{1}{2} w_{\mathrm{c}}$ and the player should agree with the oracle's prediction with probability $Q_{a}=\frac{1}{2}+\frac{1}{2}\left(w_{\mathrm{c}} / w\right)$. With these optimal wagers and strategies, Theorem 3 of [3] gives an explicit formula for the critical wager and expected value for this game:

$$
w_{\mathrm{c}}=\left(\begin{array}{c}
n-1 \\
k
\end{array}\right) / \sum_{i=0}^{k}\left(\begin{array}{l}
n \\
i
\end{array}\right)
$$

\footnotetext{
Received 21 August 2008; revision received 13 July 2009.

* Postal address: Box 166, Department of Mathematics and Computer Science, Hampden-Sydney College, HampdenSydney, VA 23943, USA.

** Postal address: Box 174, Department of Mathematics and Computer Science, Hampden-Sydney College, HampdenSydney, VA 23943, USA.

*** Current address: University of Dallas, 1845 E. Northgate Drive, Irving, TX 75062-4736, USA.

Email address: josoinach@udallas.edu
} 
and

$$
E_{n, k}=2^{n} / \sum_{i=0}^{k}\left(\begin{array}{l}
n \\
i
\end{array}\right) .
$$

Subsequent work by Ravikumar [5] demonstrated a reciprocal relationship between the lying oracle problem and the continuous version of Ulam's liar game. In that game, a questioner searches for a number in the interval $[0,1]$, chosen by a responder. The questioner's task is to find a subset of smallest measure that contains the number. The questioner asks the responder $n$ questions about the number's location in the interval, but the responder may lie up to $k$ times. This game has been studied in connection with binary search in the presence of errors in [6]. There it was shown that optimal play by both the questioner and responder yields a set of measure $\sum_{i=0}^{k}\left(\begin{array}{c}n \\ i\end{array}\right) / 2^{n}$, which is the reciprocal of the expected value of the game in the lying oracle problem.

Additionally, work in [4] described a path-guessing game in which two players traverse a directed graph. At each vertex, one player attempts to guess to which of the available vertices the other player will choose to visit next. The goal is for the first player to place wagers on which vertex the guide will choose so as to maximize his final winnings after multiple steps. This model also provides the means for the analysis of a lying oracle problem of infinite duration, as was shown in [4].

\subsection{Using a biased coin}

In this paper we investigate the variant of the lying oracle problem posed in [3], in which the oracle uses a biased coin. Specifically, the coin will land heads with probability $p$; the value of $p$ is common knowledge. Related work on games involving biased coins includes [1], where the authors used a coin-matching game to motivate their analysis, and [2], where the game of paper-scissors-stone is played with a biased coin.

The analysis of the lying oracle game is facilitated by considering the sequence of steps that the oracle and the player take. Prior to the beginning of play, the oracle and the player agree on the number of times the coin will be flipped, $n$, and the restrictions governing when or how often the oracle may lie. For example, they may agree that the oracle may lie at most $k$ times, for some $k \leq n$, or they may agree that the oracle may not lie twice in a row, or any other rule governing the oracle's behavior (see [5] for the lying oracle game played using a set of lie patterns). The game then proceeds as follows.

1. The player makes a wager $w$, given as a proportion of the player's current holdings.

2. The oracle flips the coin.

3. The oracle announces the result, but may lie about the result.

4. The player states the outcome on which to bet.

5. The player wins or loses the proportion $w$ of his fortune, depending on whether the player correctly guessed the coin's outcome.

6. The game then repeats until the sequence of plays is completed.

In order to analyze the effect that the oracle's and player's particular strategies have on the player's payoff at the conclusion of the entire game, it is helpful to consider this game in two stages. Stage one consists of steps 1-5 above, and stage two consists of multiplying the player's resulting fortune from stage one by a 'fortune multiplier'. This incorporates step 6 
by recognizing that the player's future expected payoff depends on the oracle's ability to lie in the future. Hence, if the oracle told the truth during stage one then the expected value of the player's fortune $F$ at the end of the game is expressed as $\mathrm{E}\left[F \mid F_{1}\right]=\alpha_{t} F_{1}$, where $F_{1}$ is his fortune at the end of stage one. Similarly, if the oracle lied, we have $\mathrm{E}\left[F \mid F_{1}\right]=\alpha_{\ell} F_{1}$. Thus, we can think of $\alpha_{\ell}$ and $\alpha_{t}$ as values (to the player) of a lie or truth on the part of the oracle. Of course, the specific values of $\alpha_{t}$ and $\alpha_{\ell}$ depend on the particular restrictions governing the game (e.g. the values of $n$ and $k$ ), and on the stage of play that the game has reached.

The paper is organized as follows. In Section 2 we derive the optimal strategies for both the oracle and the player in terms of $p, w, \alpha_{t}$, and $\alpha_{\ell}$. In Section 3 we apply the analysis to the original lying oracle game of $n$ flips and $k$ lies. Section 4 gives an extended example of this game. We conclude in Section 5 with some conjectures and open problems.

\section{Optimal play in stage one}

This section addresses the game that consists of a single coin toss, after which the payoff from that coin toss is multiplied by one of the values $\alpha_{t}$ or $\alpha_{\ell}$. Subsequently, we will extend the results of this section to multiple-flip games. Our goal is to derive optimal strategies for the player and the oracle in this one-step game. To simplify the presentation, we will assume that $p \geq \frac{1}{2}$ and $\alpha_{\ell}>\alpha_{t}$. There is no loss of generality here, because the complementary cases are covered by reversing the roles of heads and tails, and truth and lies. (The case where $\alpha_{\ell}=\alpha_{t}$ is trivial.)

Intuitively, three basic principles seem necessary for optimal play.

Principle 1. The oracle must make sure that his announcement gives the player no real information as to the actual outcome of the coin toss.

Principle 2. The player, knowing this, should make his decision of whether to agree or disagree with the oracle completely independently of the oracle's announcement.

Principle 3. Because the player benefits more when the oracle lies than when he tells the truth, the oracle should tell the truth with higher probability than he lies.

With the help of these principles we will derive a class of strategies for the players. We will then prove that these strategies are in fact optimal.

We begin by delineating the strategies available to the oracle and the player. First consider the oracle. The oracle's basic decision is whether to lie or tell the truth, but this decision may well depend on whether the coin landed heads or tails. Thus, the oracle actually has two separate decisions: whether to lie when the coin lands heads and whether to lie when the coin lands tails. We allow the oracle to play probabilistically, so there is a probability that the oracle tells the truth when the coin lands heads,

$$
P_{t \mid \mathrm{H}}=\operatorname{Pr}(\text { oracle tells the truth } \mid \text { coin is heads), }
$$

and a probability that the oracle tells the truth when the coin lands tails,

$$
P_{t \mid \mathrm{T}}=\operatorname{Pr}(\text { oracle tells the truth } \mid \text { coin is tails). }
$$

A strategy for the oracle in stage one is a choice of values for $P_{t \mid \mathrm{H}}$ and $P_{t \mid \mathrm{T}}$. Note that there are precisely four pure (deterministic) strategies for the oracle, corresponding to

$$
\left(P_{t \mid \mathrm{H}}, P_{t \mid \mathrm{T}}\right) \in\{(0,0),(0,1),(1,0),(1,1)\} .
$$


Note also that, by the law of total probabilities, the unconditional probability that the oracle tells the truth is

$$
P_{t}=\operatorname{Pr}(\text { oracle tells the truth })=P_{t \mid \mathrm{H}} p+P_{t \mid \mathrm{T}}(1-p) .
$$

Now consider strategies for the player. The player's basic decision is whether to agree or disagree with the oracle, but this decision may depend on whether the oracle announces heads or tails. Therefore, the player's decision is specified by the two probabilities

$$
Q_{a \mid \text { says H }}=\operatorname{Pr}(\text { player agrees with oracle | oracle announces heads) }
$$

and

$$
Q_{a \mid \text { says T }}=\operatorname{Pr}(\text { player agrees with oracle } \mid \text { oracle announces tails }) .
$$

The player's pure strategies correspond to

$$
\left(Q_{a \mid \text { says H }}, Q_{a \mid \text { says T }}\right) \in\{(0,0),(0,1),(1,0),(1,1)\} .
$$

We will denote the unconditional probability that the player agrees with the oracle by

$$
Q_{a}=\operatorname{Pr}(\text { player agrees with oracle). }
$$

In addition, the player must also choose a wager $w \in[0,1]$. We consider the player's wager to be deterministic, but still the player must choose the optimal value for the wager.

The player's fortune at the end of the one-step game (i.e. after multiplication by $\alpha_{t}$ or $\alpha_{\ell}$ ) will be denoted by $F$. Optimal play can be derived by considering the expected value of $F$ under a given player's pure strategies as a function of the other player's strategy. Looking at the oracle's pure strategies, we have the following.

1. $P_{t \mid \mathrm{H}}=0, P_{t \mid \mathrm{T}}=0$ : the oracle lies. Then

$$
\mathrm{E}[F]=\left[\left(1-Q_{a}\right)(1+w)+Q_{a}(1-w)\right] \alpha_{\ell} .
$$

2. $P_{t \mid \mathrm{H}}=0, P_{t \mid \mathrm{T}}=1$ : the oracle announces tails. Then

$$
\begin{aligned}
\mathrm{E}[F]= & \alpha_{t}(1-p)\left[(1+w) Q_{a \mid \text { says T }}+(1-w)\left(1-Q_{a \mid \text { says T }}\right)\right] \\
& +\alpha_{\ell} p\left[(1+w)\left(1-Q_{a \mid \text { says T }}\right)+(1-w) Q_{a \mid \text { says T }}\right] .
\end{aligned}
$$

3. $P_{t \mid \mathrm{H}}=1, P_{t \mid \mathrm{T}}=0$ : the oracle announces heads. Then

$$
\begin{aligned}
\mathrm{E}[F]= & \alpha_{t} p\left[(1+w) Q_{a \mid \text { says } \mathrm{H}}+(1-w)\left(1-Q_{a \mid \text { says } \mathrm{H}}\right)\right] \\
& +\alpha_{\ell}(1-p)\left[(1+w)\left(1-Q_{a \mid \text { says } \mathrm{H}}\right)+(1-w) Q_{a \mid \text { says } \mathrm{H}}\right] .
\end{aligned}
$$

4. $P_{t \mid \mathrm{H}}=1, P_{t \mid \mathrm{T}}=1$ : the oracle tells the truth. Then

$$
\mathrm{E}[F]=\left[Q_{a}(1+w)+\left(1-Q_{a}\right)(1-w)\right] \alpha_{t} .
$$

For the player, we have

1. $Q_{a \mid \text { says H }}=0, Q_{a \mid \text { says T }}=0$ : the player disagrees with the oracle. Then

$$
\mathrm{E}[F]=(1+w)\left(1-P_{t}\right) \alpha_{\ell}+(1-w) P_{t} \alpha_{t} .
$$


2. $Q_{a \mid \text { says H }}=0, Q_{a \mid \text { says T }}=1$ : the player bets on tails. Then

$$
\begin{aligned}
\mathrm{E}[F]= & (1-p)(1+w)\left[P_{t \mid \mathrm{T}} \alpha_{t}+\left(1-P_{t \mid \mathrm{T}}\right) \alpha_{\ell}\right] \\
& +p(1-w)\left[P_{t \mid \mathrm{H}} \alpha_{t}+\left(1-P_{t \mid \mathrm{H}}\right) \alpha_{\ell}\right]
\end{aligned}
$$

3. $Q_{a \mid \text { says H }}=1, Q_{a \mid \text { says T }}=0$ : the player bets on heads. Then

$$
\begin{aligned}
\mathrm{E}[F]= & p(1+w)\left[P_{t \mid \mathrm{H}} \alpha_{t}+\left(1-P_{t \mid \mathrm{H}}\right) \alpha_{\ell}\right] \\
& +(1-p)(1-w)\left[P_{t \mid \mathrm{T}} \alpha_{t}+\left(1-P_{t \mid \mathrm{T}}\right) \alpha_{\ell}\right] .
\end{aligned}
$$

4. $Q_{a \mid \text { says H }}=1, Q_{a \mid \text { says T }}=1$ : the player agrees with the oracle. Then

$$
\mathrm{E}[F]=(1+w) P_{t} \alpha_{t}+(1-w)\left(1-P_{t}\right) \alpha_{\ell} .
$$

Equilibria can be found by equating the expected values for each player, and then solving for the other player's strategy. We take a different approach here. We will use Principles 1-3 above to derive candidate optimal strategies. We will then prove that these strategies are optimal using (2)-(9).

Principle 1 states that the oracle's announcement should convey no information about the coin toss. Another way of saying this is that the oracle's announcement should be independent of the oracle's decision whether to lie or not:

$$
\operatorname{Pr}(\text { oracle tells the truth } \mid \text { oracle announces heads })=P_{t} \text {. }
$$

Expanding the conditional probability, we obtain

$$
\frac{P_{t \mid \mathrm{H}} p}{P_{t \mid \mathrm{H}} p+\left(1-P_{t \mid \mathrm{T}}\right)(1-p)}=P_{t} .
$$

Principle 2 states that the player should make his decision to agree or disagree independently of the oracle's announcement:

$$
Q_{a \mid \text { says H }}=Q_{a \mid \text { says T }}=Q_{a} \text {. }
$$

Principle 3 states that the oracle should tell the truth with higher probability than he lies. But how much higher? Perhaps the simplest choice is to make the probability of telling the truth (or lying) inversely proportional to the value of telling the truth (or lying):

$$
P_{t}=\frac{\alpha_{t}^{-1}}{\alpha_{t}^{-1}+\alpha_{\ell}^{-1}}=\frac{\alpha_{\ell}}{\alpha_{\ell}+\alpha_{t}} .
$$

We shall take (10)-(12) as the starting points for our derivation of candidate optimal strategies.

Solving (1) and (10) simultaneously in light of (12) gives

$$
P_{t \mid \mathrm{H}}=\frac{\alpha_{\ell}}{\alpha_{\ell}+\alpha_{t}} \frac{\alpha_{\ell}-\alpha_{t}(1-p) / p}{\alpha_{\ell}-\alpha_{t}}
$$

and

$$
P_{t \mid \mathrm{T}}=\frac{\alpha_{\ell}}{\alpha_{\ell}+\alpha_{t}} \frac{\alpha_{\ell}-\alpha_{t} p /(1-p)}{\alpha_{\ell}-\alpha_{t}} .
$$


Note that, when $p=\frac{1}{2}$, we have $P_{t \mid \mathrm{H}}=P_{t \mid \mathrm{T}}=\alpha_{\ell} /\left(\alpha_{\ell}+\alpha_{t}\right)$, while $p=\alpha_{\ell} /\left(\alpha_{\ell}+\alpha_{t}\right)$ gives $P_{t \mid \mathrm{H}}=1$ and $P_{t \mid \mathrm{T}}=0$. Therefore, (13) and (14) are valid probabilities as long as $p$ satisfies

$$
\frac{1}{2} \leq p \leq \frac{\alpha_{\ell}}{\alpha_{\ell}+\alpha_{t}} .
$$

Accordingly, we restrict to this case first. What is the player's expected fortune if the oracle adopts the strategy given by (13) and (14)? Substituting (13) and (14) into (6)-(9), we see that, for each of the player's pure strategies, we have

$$
\mathrm{E}[F]=\frac{2 \alpha_{\ell} \alpha_{t}}{\alpha_{\ell}+\alpha_{t}} \equiv H
$$

Note that $H$ is the harmonic mean of the values $\alpha_{t}$ and $\alpha_{\ell}$. Since any mixed strategy is a convex combination of the pure strategies, it follows that the player's expected fortune under any strategy whatsoever is still equal to $H$, provided that the oracle uses the strategy given by (13) and (14). Moreover, it is straightforward to show that if the oracle uses any other strategy then the player can find a strategy that makes his expected fortune strictly greater than $H$. (For instance, if the oracle chooses a strategy that makes $P_{t}<\alpha_{\ell} /\left(\alpha_{\ell}+\alpha_{t}\right)$ then the player could use his pure strategy (6) with $w=1$, which yields $\mathrm{E}[F]=2\left(1-P_{t}\right) \alpha_{\ell}>H$. Other cases are handled similarly.) This proves that (13) and (14) constitute an equilibrium strategy for the oracle.

Still assuming (15), now consider the situation from the player's point of view: he knows that the coin is heads with probability $p$, while the oracle tells the truth with probability $P_{t}=$ $\alpha_{\ell} /\left(\alpha_{\ell}+\alpha_{t}\right) \geq p$ (because (15) holds). Thus, the oracle is at least as reliable as the coin in this case, and it would seem that the player could not do any better than to agree with the oracle. This along with (11) gives

$$
Q_{a \mid \text { says H }}=Q_{a \mid \text { says T }}=Q_{a}=1 \text {. }
$$

The player also needs to decide on a wager. Here we adopt the usual procedure of choosing a wager such that the player's expected fortune is the same whether or not the oracle lies. An easy calculation gives

$$
w=\frac{\alpha_{\ell}-\alpha_{t}}{\alpha_{\ell}+\alpha_{t}}
$$

Substituting (16) and (17) into the oracle's pure strategies, (2)-(5), we find that not only does (2) continue to hold for all the pure strategies, but in fact the player's fortune is nonrandom:

$$
F=H \quad \text { with probability } 1 .
$$

Since this is the case for each of the oracle's pure strategies, it must hold for any mixed strategy as well. Thus, by adopting the strategy given by (16) and (17), the player can guarantee himself a fortune of $H$, no matter which strategy the oracle adopts. But all that is required for an equilibrium is that the expected value of the player's fortune equals the harmonic mean. This leads to a wider class of equilibrium strategies for the player. Necessary and sufficient conditions for an equilibrium strategy are found by equating (2)-(5) under assumption (15). This is a straightforward exercise that results in a class of mixed strategies for the player. To describe them, denote the value given by (17) as the critical wager $w_{\mathrm{c}}$ :

$$
w_{\mathrm{c}}=\frac{\alpha_{\ell}-\alpha_{t}}{\alpha_{\ell}+\alpha_{t}}
$$


The equilibrium strategies for the player are characterized by a wager $w$ that is at least as large as the critical wager,

$$
w \in\left[w_{\mathrm{c}}, 1\right]
$$

and a probability of agreement given by

$$
Q_{a \mid \text { says H }}=Q_{a \mid \text { says T }}=\frac{1}{2}\left(1+\frac{w_{\mathrm{c}}}{w}\right) .
$$

Substituting (18) and (19) into (2)-(5), we see that the expected value of the player's fortune under all the oracle's pure strategies is again equal to $H$. It can also be shown that if the player uses any strategy other than those given by (18) and (19), then the oracle has a strategy that reduces the player's expected fortune below $H$. (In particular, if $Q_{a \mid \text { says H }} \neq Q_{a \mid \text { says T }}$ then one of (3) or (4) will be less than $H$, while if $\left(2 Q_{a}-1\right) w \neq w_{\mathrm{c}}$ then either (2) or (5) will be less than $H$.) This shows that (18) and (19) constitute the equilibrium strategies for the player in the case (15).

Note that if the player wagers the critical amount $w_{\mathrm{c}}$ then this is the same pure strategy derived above. Wagers greater than the critical amount give optimal mixed strategies. In the extreme case, when $w=1$, the optimal probability of agreement is

$$
Q_{a \mid \text { says H }}=Q_{a \mid \text { says T }}=\frac{1}{2}\left(1+w_{\mathrm{c}}\right)=\frac{\alpha_{\ell}}{\alpha_{\ell}+\alpha_{t}},
$$

which is the same as the oracle's probability of telling the truth. An interesting aspect of case (15) is that it actually benefits the oracle to be more reliable than the coin. This in turn entails another interesting fact: the player's optimal strategies do not depend on $p$ so long as (15) holds.

The remaining case is

$$
p>\frac{\alpha_{\ell}}{\alpha_{\ell}+\alpha_{t}}
$$

Note that in the boundary case when $p=\alpha_{\ell} /\left(\alpha_{\ell}+\alpha_{t}\right)$ the oracle is announcing heads with probability 1 by (13) and (14). Thus, the oracle and the coin are equally reliable at this boundary case. If (20) holds, however, then the oracle can no longer be more reliable than the coin and still avoid giving information to the player. But he can remain equally as reliable as the coin, and still avoid giving information to the player, by simply continuing to announce heads with probability 1 . This is in fact optimal for the oracle. To see this, note that if the player bets his entire fortune on heads regardless of what the oracle announces then his expected fortune is at least $2 p \alpha_{t}$ (recall that $\alpha_{t}<\alpha_{\ell}$ ), with equality if and only if the oracle announces heads with probability 1 . So the oracle cannot hope to reduce $\mathrm{E}[F]$ below this value. Can the player do any better than an expected fortune of $2 p \alpha_{t}$ ? In other words, could it possibly benefit the player to bet on tails with positive probability? We claim not. To see this, let $Q_{\mathrm{T}}$ be the unconditional probability that the player bets on tails. Let the oracle's strategy continue to be to announce heads with probability 1 . Then the player's expected fortune is

$$
\begin{aligned}
\mathrm{E}[F]= & (1-p)\left[(1+w) Q_{\mathrm{T}}+(1-w)\left(1-Q_{\mathrm{T}}\right)\right] \alpha_{\ell} \\
& +p\left[(1+w)\left(1-Q_{\mathrm{T}}\right)+(1-w) Q_{\mathrm{T}}\right] \alpha_{t} \\
= & w\left(1-2 Q_{\mathrm{T}}\right)\left[p \alpha_{t}-(1-p) \alpha_{\ell}\right]+\left[p \alpha_{t}+(1-p) \alpha_{\ell}\right] .
\end{aligned}
$$

Note that the first term in square brackets is positive because of (20). Therefore, (21) is uniquely maximized when $w=1$ and $Q_{\mathrm{T}}=0$, and the maximum value is $2 p \alpha_{t}$. In other words, the 
oracle can limit the player's expected fortune to $2 p \alpha_{t}$ by always announcing heads, and a positive probability of betting on tails only decreases the player's expected fortune against this strategy. This proves our claim. We have now shown that optimal play when $p>\alpha_{\ell} /\left(\alpha_{\ell}+\alpha_{t}\right)$ is given by

$$
P_{t \mid \mathrm{H}}=1 \quad \text { and } \quad P_{t \mid \mathrm{T}}=0
$$

and

$$
Q_{a \mid \text { says H }}=1, \quad Q_{a \mid \text { says T }}=0, \quad \text { and } \quad w=1 .
$$

We summarize this discussion in the following theorem.

Theorem 1. (Optimal play.) Assume without loss of generality that $p \geq \frac{1}{2}$ and $\alpha_{\ell}>\alpha_{t}$. The optimal strategy for the oracle in stage one is given by

$$
P_{t \mid \mathrm{H}}= \begin{cases}\frac{\alpha_{\ell}}{\alpha_{\ell}+\alpha_{t}} \frac{\alpha_{\ell}-\alpha_{t}(1-p) / p}{\alpha_{\ell}-\alpha_{t}} & \text { if } p \leq \frac{\alpha_{\ell}}{\alpha_{t}+\alpha_{\ell}} \\ 1 & \text { otherwise }\end{cases}
$$

and

$$
P_{t \mid \mathrm{T}}= \begin{cases}\frac{\alpha_{\ell}}{\alpha_{\ell}+\alpha_{t}} \frac{\alpha_{\ell}-\alpha_{t} p /(1-p)}{\alpha_{\ell}-\alpha_{t}} & \text { if } p \leq \frac{\alpha_{\ell}}{\alpha_{t}+\alpha_{\ell}}, \\ 0 & \text { otherwise. }\end{cases}
$$

For the player, if $p \leq \alpha_{\ell} /\left(\alpha_{\ell}+\alpha_{t}\right)$ then the optimal strategy is given by

$$
w \geq w_{\mathrm{c}} \text { and } Q_{a \mid \text { says } \mathrm{H}}=Q_{a \mid \text { says } \mathrm{T}}=\frac{1}{2}\left(1+\frac{w_{\mathrm{c}}}{w}\right),
$$

while if $p>\alpha_{\ell} /\left(\alpha_{\ell}+\alpha_{t}\right)$ then

$$
w=1, \quad Q_{a \mid \text { says } \mathrm{H}}=1, \quad \text { and } Q_{a \mid \text { says } \mathrm{T}}=0
$$

is optimal, where $w_{\mathrm{c}}=\left(\alpha_{\ell}-\alpha_{t}\right) /\left(\alpha_{\ell}+\alpha_{t}\right)$ is the player's critical wager. Under optimal play, the player's fortune satisfies

$$
\mathrm{E}[F]= \begin{cases}\frac{2 \alpha_{t} \alpha_{\ell}}{\alpha_{t}+\alpha_{\ell}} & \text { if } p \leq \frac{\alpha_{\ell}}{\alpha_{t}+\alpha_{\ell}} \\ 2 p \alpha_{t} & \text { otherwise. }\end{cases}
$$

\section{The analysis of the $n$ flip, $k$ lie game}

We now use the above analysis to solve a problem posed in [3]. As in the original lying oracle problem, the oracle will flip a coin $n$ times and may lie at most $k$ times, with $k \leq n$, but as above, the oracle will use a biased coin, where the coin will land on heads with probability $p>\frac{1}{2}$. If the player and the oracle play optimally, then define $E_{n, k}$ to be the expected payoff for the game of $n$ flips and $k$ lies.

Under these conditions, if the game begins with $n$ flips and $k$ lies, then $\alpha_{t}=E_{n-1, k}$ and $\alpha_{l}=E_{n-1, k-1}$, as these represent the expected payoffs for the game with one fewer flip and either the same number of lies (the oracle told the truth) or one less lie (the oracle lied about the outcome). The task is to specify these expected payoffs in terms of $n, k$, and $p$. 
We first recall from the previous results that $E_{n, 0}=2^{n}$ and that $E_{n, n}=(2 p) E_{n-1, n-1}=$ $(2 p)^{n}$. Furthermore, Theorem 1 asserts that if

$$
p<\frac{\alpha_{l}}{\alpha_{t}+\alpha_{l}}
$$

then the player's expected payoff under optimal play is

$$
E_{n, k}=\frac{2 E_{n-1, k} E_{n-1, k-1}}{E_{n-1, k}+E_{n-1, k-1}},
$$

while if

$$
p \geq \frac{\alpha_{l}}{\alpha_{t}+\alpha_{l}}
$$

then the player will bet on heads and will have an expected payoff of

$$
E_{n, k}=(2 p) E_{n-1, k}=(2 p)^{n} .
$$

It is convenient to work with the reciprocals of our expected payoffs $E_{n, k}$. We therefore summarize the above results as follows:

$$
E_{n, k}^{-1}= \begin{cases}\frac{1}{(2 p)^{n}}, & p \geq \frac{\alpha_{l}}{\alpha_{t}+\alpha_{l}} \\ \frac{1}{2}\left(E_{n-1, k}^{-1}+E_{n-1, k-1}^{-1}\right), & p<\frac{\alpha_{l}}{\alpha_{t}+\alpha_{l}} .\end{cases}
$$

\subsection{The functions $g_{n, k}(x)$ and $f_{n, k}(x)$}

Since the value of $E_{n, k}^{-1}$ is defined recursively, we can define functions $g_{n, k}(x)$ recursively such that the reciprocals $E_{n, k}^{-1}$ have the following form:

$$
E_{n, k}^{-1}=\frac{g_{n, k}(p)}{(2 p)^{n}} .
$$

Accordingly, we define

$$
g_{n, k}(x)= \begin{cases}x^{n}, & k=0, \\ 1, & k=n, \\ \min \left(1, x\left(g_{n-1, k}(x)+g_{n-1, k-1}(x)\right)\right), & 0<k<n .\end{cases}
$$

Lemma 1. For all $n \geq 0$, all $0 \leq k \leq n$, and all $p \in\left[\frac{1}{2}, 1\right]$,

$$
E_{n, k}=\frac{(2 p)^{n}}{g_{n, k}(p)} .
$$

Proof. Let $p \in\left[\frac{1}{2}, 1\right]$. If $k=0$ then the oracle cannot lie, so the player's expected fortune is

$$
E_{n, 0}=2^{n}=\frac{(2 p)^{n}}{p^{n}}=\frac{(2 p)^{n}}{g_{n, 0}(p)} .
$$

If $k=n$ then the oracle can lie every time. The player will always bet everything on the coin (heads), and so the player's expected fortune is

$$
E_{n, n}=(2 p)^{n}=\frac{(2 p)^{n}}{g_{n, n}(p)} .
$$


In the third case, suppose that $0<k<n$. Then the player will either bet on the coin or agree with the oracle, whichever one produces the greater expected payoff. If the player bets on the coin, then betting everything on heads is optimal and the player's expected payoff is

$$
E_{n, k}=(2 p) E_{n-1, k}=(2 p)^{n} .
$$

If the player agrees with the oracle then

$$
E_{n, k}=\frac{2 E_{n-1, k} E_{n-1, k-1}}{E_{n-1, k}+E_{n-1, k-1}} .
$$

Since the player chooses the strategy with the greater expected payoff, it follows that

$$
E_{n, k}=\max \left((2 p)^{n}, \frac{2 E_{n-1, k} E_{n-1, k-1}}{E_{n-1, k}+E_{n-1, k-1}}\right)
$$

and

$$
\begin{aligned}
E_{n, k}^{-1} & =\min \left(\frac{1}{(2 p)^{n}}, \frac{1}{2}\left(E_{n-1, k}^{-1}+E_{n-1, k-1}^{-1}\right)\right) \\
& =\min \left(\frac{1}{(2 p)^{n}}, \frac{1}{2}\left(\frac{g_{n-1, k}(p)+g_{n-1, k-1}(p)}{(2 p)^{n-1}}\right)\right) \quad \text { (by induction) } \\
& =\min \left(\frac{1}{(2 p)^{n}}, \frac{p\left(g_{n-1, k}(p)+g_{n-1, k-1}(p)\right)}{(2 p)^{n}}\right) \\
& =\frac{\min \left(1, p\left(g_{n-1, k}(p)+g_{n-1, k-1}(p)\right)\right)}{(2 p)^{n}} \\
& =\frac{g_{n, k}(p)}{(2 p)^{n}}
\end{aligned}
$$

Thus, in all cases

$$
E_{n, k}=\frac{(2 p)^{n}}{g_{n, k}(p)}
$$

Corollary 1. For all $n \geq 0$, all $0 \leq k \leq n$, and all $p \in\left[\frac{1}{2}, 1\right]$,

$$
g_{n, k}(p)= \begin{cases}1, & p \geq \alpha_{l} /\left(\alpha_{t}+\alpha_{l}\right), \\ p\left(g_{n-1, k}(p)+g_{n-1, k-1}(p)\right), & p<\alpha_{l} /\left(\alpha_{t}+\alpha_{l}\right) .\end{cases}
$$

Since the expected payoff to the player depends on how $p$ compares to

$$
\frac{\alpha_{l}}{\alpha_{t}+\alpha_{l}}=\frac{E_{n-1, k-1}}{E_{n-1, k}+E_{n-1, k-1}}
$$

we would like to find a way to compare $p$ involving the functions $g_{n, k}(x)$. We accomplish this in the next lemma.

Lemma 2. For all $n \geq 1$ and all $0 \leq k \leq n$, the player should bet on heads (regardless of the oracle's prediction) if and only if

$$
p g_{n-1, k-1}(p)+p-1 \geq 0 .
$$


Proof. The optimal strategy for the player is to bet on heads regardless of the oracle's prediction if and only if $p \geq \alpha_{l} /\left(\alpha_{t}+\alpha_{l}\right)$. So, suppose that $p \geq \alpha_{l} /\left(\alpha_{t}+\alpha_{l}\right)$. Then

$$
\begin{aligned}
p & \geq \frac{E_{n-1, k-1}}{E_{n-1, k}+E_{n-1, k-1}}, \\
p\left(E_{n-1, k}+E_{n-1, k-1}\right) & \geq E_{n-1, k-1}, \\
p E_{n-1, k} & \geq(1-p) E_{n-1, k-1}, \\
p E_{n-1, k-1}^{-1} & \geq(1-p) E_{n-1, k}^{-1}, \\
p g_{n-1, k-1}(p) & \geq(1-p) g_{n-1, k}(p), \\
p g_{n-1, k-1}(p)-(1-p) g_{n-1, k}(p) & \geq 0 .
\end{aligned}
$$

By assumption, $p \geq \alpha_{l} /\left(\alpha_{t}+\alpha_{l}\right)$, so that $g_{n, k}(x)=1$, so that $g_{n-1, k}(x)=1$ as well. Thus, we have $p g_{n-1, k-1}(p)+p-1 \geq 0$, as desired.

Conversely, if $p<\alpha_{l} /\left(\alpha_{t}+\alpha_{l}\right)$ then the above computation yields

$$
0>p g_{n-1, k-1}(p)+(p-1) g_{n-1, k}(p) .
$$

However, by (22), $g_{n-1, k}(p) \leq 1$. Therefore,

$$
0>p g_{n-1, k-1}(p)+(p-1) g_{n-1, k}(p) \geq p g_{n-1, k-1}(p)+(p-1),
$$

as desired. Therefore, let us define

$$
f_{n, k}(x)=x g_{n-1, k-1}(x)+x-1 .
$$

Lemma 2 may thus be restated by saying the player should bet on heads if and only if $f_{n, k}(p) \geq$ 0 . The following two lemmata describe some of the basic properties of the function $g_{n, k}(x)$.

Lemma 3. Let $g_{n, k}(x)$ be defined as in (22). Then $g_{n, k}(x) \leq g_{n-1, k}(x)$ for $\frac{1}{2} \leq x \leq 1,0 \leq$ $k \leq n$, and $n \geq k+1$.

Proof. The proof is by induction on $n$ and $k$. We need to establish two base cases: $k=0$ and $k=1$, each for all $n \geq k+1$.

First, when $k=0$, it is clear that $g_{n, 0}(x) \leq g_{n-1,0}(x)$ for all $x \in\left[\frac{1}{2}, 1\right]$ because $g_{n, 0}(x)=x^{n}$. (No induction necessary.)

Now let $k=1$ and begin with $n=2$. It has been shown above that $g_{2,1}(x) \leq g_{1,1}(x)$ for all $x \in\left[\frac{1}{2}, 1\right]$. Now suppose that $g_{r, 1}(x) \leq g_{r-1,1}(x)$ for all $x \in\left[\frac{1}{2}, 1\right]$ and some $r \geq 2$. Then

$$
\begin{aligned}
g_{r+1,1}(x) & =\min \left(1, x\left(g_{r, 1}(x)+g_{r, 0}(x)\right)\right) \\
& \leq \min \left(1, x\left(g_{r-1,1}(x)+g_{r-1,0}(x)\right)\right) \\
& =g_{r, 1}(x) .
\end{aligned}
$$

Thus, $g_{n, 1}(x) \leq g_{n-1,1}(x)$ for all $x \in\left[\frac{1}{2}, 1\right]$ and all $n \geq 2$.

We now proceed by induction on $k$. Suppose that $g_{n, s}(x) \leq g_{n-1, s}(x)$ for all $x \in\left[\frac{1}{2}, 1\right]$, all $n \geq s+1$, and some $s \geq 1$. We will show that $g_{n, s+1}(x) \leq g_{n-1, s+1}(x)$ for all $x \in\left[\frac{1}{2}, 1\right]$ and all $n \geq s+2$. This will complete the proof. First, $g_{s+1, s+1}(x)=1$ and

$$
\begin{aligned}
g_{s+2, s+1}(x) & =\min \left(1, x\left(g_{s+1, s+1}(x)+g_{s+1, s}(x)\right)\right) \\
& =\min \left(1, x\left(1+g_{s+1, s}(x)\right)\right) \\
& \leq 1 \\
& =g_{s+1, s+1}(x) .
\end{aligned}
$$


So the statement is true when $n=s+2$. Now suppose that it is true when $n=r$ for some $r \geq s+2$. That is, suppose that

$$
g_{r, s+1}(x) \leq g_{r-1, s+1}(x)
$$

for all $x \in\left[\frac{1}{2}, 1\right]$. Then

$$
\begin{aligned}
g_{r+1, s+1}(x) & =\min \left(1, x\left(g_{r, s+1}(x)+g_{r, s}(x)\right)\right) \\
& \leq \min \left(1, x\left(g_{r-1, s+1}(x)+g_{r-1, s}(x)\right)\right) \\
& =g_{r, s+1}(x) .
\end{aligned}
$$

This completes the proof.

Lemma 4. For every $k \geq 0$ and $x \in\left[\frac{1}{2}, 1\right)$,

$$
\lim _{n \rightarrow \infty} g_{n, k}(x)=0 \text {. }
$$

Proof. By definition (22) of $g_{n, k}(x)$ we have

$$
g_{n, k}(x) \leq x\left(g_{n-1, k}(x)+g_{n-1, k-1}(x)\right) .
$$

Exploiting the similarity between this inequality and Pascal's formula,

$$
\left(\begin{array}{l}
n \\
k
\end{array}\right)=\left(\begin{array}{c}
n-1 \\
k
\end{array}\right)+\left(\begin{array}{l}
n-1 \\
k-1
\end{array}\right),
$$

it is easy to show by induction that

$$
g_{n, k}(x) \leq \sum_{j=0}^{k}\left(\begin{array}{c}
n-k-1+j \\
n-k-1
\end{array}\right) x^{n-k+j} .
$$

It then follows, for fixed $k, x$, and $j$, that

$$
\lim _{n \rightarrow \infty}\left(\begin{array}{c}
n-k-1+j \\
n-k-1
\end{array}\right) x^{n-k+j}=0,
$$

because

$$
\left(\begin{array}{c}
n-k-1+j \\
n-k-1
\end{array}\right)
$$

is a polynomial in $n$ while $x^{n-k+j}$ is an exponential function in $n$, with base $x<1$. Therefore,

$$
\lim _{n \rightarrow \infty} g_{n, k}(x)=0 \text {. }
$$

Therefore, according to (23), for fixed $k$ and $p$, we have $f_{n, k}(p) \leq 0$ for sufficiently large $n$. Let $m_{k}$ be the largest value of $n$ such that $f_{n, k}(p)>0$, which by Lemma 2 implies that the player should play a mixed strategy given by Theorem 1 for all $n>m_{k}$. For $n \leq m_{k}$, the player should bet on heads and the oracle should announce heads.

It is clear from the definitions of $g_{n, k}(x)$ and $f_{n, k}(x)$ that they are increasing functions on the interval $\left[\frac{1}{2}, 1\right]$. It is also clear that $g_{n, k}\left(\frac{1}{2}\right) \leq 1$ and $g_{n, k}(1)=1$, and, therefore, $f_{n, k}\left(\frac{1}{2}\right) \leq 0$ and $f_{n, k}(1)=1$. Therefore, each function $f_{n, k}(x)$ has a unique real root in the interval $\left[\frac{1}{2}, 1\right]$. Denote this root by $p_{n, k}$. 
Lemma 5. Let $p_{n, k}$ and $m_{k}$ be defined as above. Then $p_{n+1, k} \geq p_{n, k}$ for all $n \geq 1,0 \leq k \leq n$, and $m_{k+1}>m_{k}$.

Proof. To prove that $p_{n+1, k} \geq p_{n, k}$, we evaluate $f_{n+1, k}\left(p_{n, k}\right)$ :

$$
\begin{aligned}
f_{n+1, k}\left(p_{n, k}\right) & =p_{n, k} g_{n, k-1}\left(p_{n, k}\right)+p_{n, k}-1 \\
& =p_{n, k} g_{n, k-1}\left(p_{n, k}\right)-p_{n, k} g_{n-1, k-1}\left(p_{n, k}\right)+f_{n, k}\left(p_{n, k}\right) \\
& =p_{n, k}\left(g_{n, k-1}\left(p_{n, k}\right)-g_{n-1, k-1}\left(p_{n, k}\right)\right) .
\end{aligned}
$$

This last quantity is nonpositive, since according to Lemma $3, g_{n, k}(x) \leq g_{n-1, k}(x)$ for all $x \in\left[\frac{1}{2}, 1\right]$. Thus, by the monotonicity of $f_{n+1, k}(x)$, we have $p_{n+1, k} \geq p_{n, k}$.

Similarly, to prove that $m_{k+1}>m_{k}$, we first note that, by the definition of $m_{k}, f_{m_{k}, k}(p)>0$ and $f_{m_{k}+1, k}(p) \leq 0$, so that $g_{m_{k}, k}(p)=1$. We now evaluate $f_{m_{k}+1, k+1}(p)$ :

$$
f_{m_{k}+1, k+1}(p)=p g_{m_{k}, k}(p)+p-1=p \cdot 1+p-1=2 p-1>0 .
$$

Therefore, $m_{k+1}>m_{k}$.

This lemma implies that each $m_{k}$ is unique and the sequence $\left\{m_{k}\right\}$ is a strictly increasing sequence.

\subsection{Closed-form expressions for $g_{n, k}(x)$ and $f_{n, k}(x)$}

We first motivate the way the functions will be written by developing several special cases. We begin by noting that $g_{n, 0}(x)=x^{n}$, and we proceed to develop expressions for the functions when $k=1$ and $k=2$.

When $k=1$, then

$$
f_{n, 1}(x)=x g_{n-1,0}(x)+x-1=x^{n}+x-1,
$$

by the definition of $f_{n, k}(x)$ given by (23). Furthermore, when $n>m_{1}$, we use the recursive formula for $g_{n, k}(x)$ given by (22) to express $g_{n, 1}(x)$ in closed form:

$$
\begin{aligned}
g_{n, 1}(x) & =x\left(g_{n-1,1}(x)+g_{n-1,0}(x)\right) \\
& \left.=x^{2}\left(g_{n-2,1}(x)+g_{n-2,0}(x)\right)+x g_{n-1,0}(x)\right) \\
& =\cdots \\
& =x^{n-m_{1}}\left(1+g_{m_{1}, 0}(x)\right)+x^{n-m_{1}-1} g_{m_{1}+1,0}(x)+\cdots+x g_{n-1,0}(x) \\
& =\left(\sum_{i=1}^{n-m_{1}} x^{i} g_{n-i, 0}(x)\right)+x^{n-m_{1}} \\
& =\left(\sum_{i=1}^{n-m_{1}} x^{i} x^{n-i}\right)+x^{n-m_{1}} \\
& =\left(\sum_{i=1}^{n-m_{1}} x^{n}\right)+x^{n-m_{1}} \\
& =\left(n-m_{1}\right) x^{n}+x^{n-m_{1}} .
\end{aligned}
$$


We now proceed to the $k=2$ case. Using (23), we find that

$$
\begin{aligned}
f_{n, 2}(x) & =x g_{n-1,1}(x)+x-1 \\
& =x\left(\left(n-1-m_{1}\right) x^{n-1}+x^{n-1-m_{1}}\right)+x-1 \\
& =\left(n-1-m_{1}\right) x^{n}+x^{n-m_{1}}+x-1 .
\end{aligned}
$$

Next, when $n>m_{2}$, we calculate $g_{n, 2}(x)$ using the recursive formula (22):

$$
\begin{aligned}
g_{n, 2}(x) & =x\left(g_{n-1,2}(x)+g_{n-1,1}(x)\right) \\
& =x^{2}\left(g_{n-2,2}(x)+g_{n-2,1}(x)\right)+x g_{n-1,1}(x) \\
& =\cdots \\
& =x^{n-m_{2}}\left(1+g_{m_{2}, 1}(x)\right)+x^{n-m_{2}-1} g_{m_{2}+1,1}(x)+\cdots+x g_{n-1,1}(x) \\
& =\left(\sum_{i=1}^{n-m_{2}} x^{i} g_{n-i, 1}(x)\right)+x^{n-m_{2}} \\
& =\left(\sum_{i=1}^{n-m_{2}} x^{i}\left(\left(\sum_{j=1}^{n-i-m_{1}} x^{n-i}\right)+x^{n-i-m_{1}}\right)\right)+x^{n-m_{2}} \\
& =\sum_{i=1}^{n-m_{2}} \sum_{j=1}^{n-i-m_{1}} x^{n}+\sum_{i=1}^{n-m_{2}} x^{n-m_{1}}+x^{n-m_{2}} .
\end{aligned}
$$

Generalizing from the above, we now state the closed formula for the general case.

Theorem 2. For $n>m_{k}$,

$$
\begin{aligned}
g_{n, k}(x)= & \sum_{i_{1}=1}^{n-m_{k}} \sum_{i_{2}=1}^{n-i_{1}-m_{k-1}} \cdots \sum_{i_{n}=1}^{n-\left(\sum_{j=1}^{n-1} i_{j}\right)-m_{1}} x^{n} \\
& +\sum_{i_{1}=1}^{n-m_{k}} \sum_{i_{2}=1}^{n-i_{1}-m_{k-1}} \cdots \sum_{i_{n-1}=1}^{n-\left(\sum_{j=1}^{n-2} i_{j}\right)-m_{2}} x^{n-m_{1}} \\
& +\cdots+\sum_{i_{1}=1}^{n-m_{k}} x^{n-m_{k-1}}+x^{n-m_{k}}
\end{aligned}
$$

and

$$
\begin{aligned}
f_{n, k}(x)= & \sum_{i_{1}=1}^{n-1-m_{k-1}} \sum_{i_{2}=1}^{n-1-i_{1}-m_{k-2}} \cdots \sum_{i_{n-1}=1}^{n-1-\left(\sum_{j=1}^{n-2} i_{j}\right)-m_{1}} x^{n} \\
& +\sum_{i_{1}=1}^{n-1-m_{k-1}} \sum_{i_{2}=1}^{n-1-i_{1}-m_{k-2}} \cdots \sum_{i_{n-2}=1}^{n-1-\left(\sum_{j=1}^{n-3} i_{j}\right)-m_{2}} x^{n-m_{1}} \\
& +\cdots+\sum_{i_{1}=1}^{n-1-m_{k-1}} x^{n-m_{k-2}}+x^{n-m_{k-1}}+x-1 .
\end{aligned}
$$


Proof. The proof is a rather laborious but straightforward induction. The proofs of the base cases for both functions are given in the above discussion. Furthermore, the inductive step simply involves simplifying the expressions

$$
\left(\sum_{i_{1}=1}^{n-m_{k}} x^{i_{1}} g_{n-i_{1}, k-1}(x)\right)+x^{n-m_{k}}
$$

and

$$
x g_{n-1, k-1}(x)+x-1
$$

using the formulae for $g_{n, k}(x)$ and $f_{n, k}(x)$ given by (22) and (23), respectively. The details are left to the reader.

We conclude this section with the analysis of the special case when $k=n-1$. In this case we begin by noting that $g_{n, n}(x)=1$. We then use the recursive formula (22):

$$
g_{n, n-1}(x)=x\left(1+g_{n-1, n-2}(x)\right)=x+x^{2}\left(1+g_{n-2, n-3}(x)\right)=\cdots=\sum_{i=1}^{n} x^{i} .
$$

From (23) we thus obtain

$$
f_{n, n-1}(x)=x g_{n-1, n-2}(x)+x-1=x\left(\sum_{i=1}^{n-1} x^{i}\right)+x-1=\left(\sum_{i=1}^{n} x^{i}\right)-1 .
$$

\section{An extended example}

Suppose that the player and the oracle decide to play the game $G_{20,4}$ using a biased coin with bias $p=\frac{2}{3}$. What is the player's strategy and expected payoff?

We begin with the case in which $k=1$ and find the values of the functions

$$
f_{n, 1}(x)=x g_{n-1,0}(x)+x-1=x^{n}+x-1
$$

at $x=p$. We compute

$$
\begin{aligned}
& f_{1,1}(p)=2 p-1>0, \\
& f_{2,1}(p)=p^{2}+p-1>0, \\
& f_{3,1}(p)=p^{3}+p-1<0 .
\end{aligned}
$$

This implies that $m_{1}=2$ and, thus, allows us to calculate $g_{n, 1}(x)$ for $n \geq 3$ :

$$
g_{n, 1}(x)=\sum_{i=1}^{n-2} x^{n}+x^{n-2}=(n-2) x^{n}+x^{n-2} .
$$

We now continue with the case in which $k=2, n \geq 3$, and find the values of the functions

$$
f_{n, 2}(x)=x g_{n-1,1}(x)+x-1=(n-3) x^{n}+x^{n-2}+x-1
$$

at $x=p$. We compute

$$
\begin{aligned}
f_{3,2}(p) & =2 p-1>0, \\
& \vdots \\
f_{7,2}(p) & =4 p^{7}+p^{5}+p-1>0, \\
f_{8,2}(p) & =5 p^{8}+p^{6}+p-1<0 .
\end{aligned}
$$


We thus find that $m_{2}=7$, which allows us to calculate $g_{n, 2}(x)$ for $n \geq 8$ :

$$
\begin{aligned}
g_{n, 2}(x) & =\sum_{i=1}^{n-7} \sum_{j=1}^{n-i-2} x^{n}+\sum_{i=1}^{n-7} x^{n-2}+x^{n-7} \\
& =\sum_{i=1}^{n-7}(n-i-2) x^{n}+(n-7) x^{n-2}+x^{n-7} .
\end{aligned}
$$

We continue with the case in which $k=3, n \geq 8$, in this fashion and evaluate

$$
f_{n, 3}(x)=x g_{n-1,2}(x)+x-1=\left(\sum_{i=1}^{n-8}(n-i-3)\right) x^{n}+(n-8) x^{n-2}+x^{n-7}+x-1
$$

at $x=p$. We compute

$$
\begin{aligned}
f_{8,3}(p) & =2 p-1>0 \\
& \vdots \\
f_{12,3}(p) & =26 p^{12}+4 p^{10}+p^{5}+p-1>0, \\
f_{13,3}(p) & =35 p^{13}+5 p^{11}+p^{6}+p-1<0 .
\end{aligned}
$$

Thus, we have $m_{3}=12$, and we can find $g_{n, 3}(x)$ for $n \geq 13$ :

$$
g_{n, 3}(x)=\sum_{i_{1}=1}^{n-12} \sum_{i_{2}=1}^{n-i_{1}-7} \sum_{i_{3}=1}^{n-i_{1}-i_{2}-2} x^{n}+\sum_{i_{1}=1}^{n-12} \sum_{i_{2}=1}^{n-i_{1}-7} x^{n-2}+\sum_{i_{1}=1}^{n-12} x^{n-7}+x^{n-12} .
$$

Finally, we analyze the case in which $k=4, n \geq 13$, and evaluate

$$
f_{n, 4}(x)=\sum_{i_{1}=1}^{n-13} \sum_{i_{2}=1}^{n-i_{1}-8} \sum_{i_{3}=1}^{n-i_{1}-i_{2}-3} x^{n}+\sum_{i_{1}=1}^{n-13} \sum_{i_{2}=1}^{n-i_{1}-8} x^{n-2}+\sum_{i_{1}=1}^{n-13} x^{n-7}+x^{n-12}+x-1
$$

at $x=p$. We compute

$$
\begin{aligned}
f_{13,4}(p) & =2 p-1>0, \\
& \vdots \\
f_{18,4}(p) & =285 p^{18}+35 p^{16}+5 p^{11}+p^{6}+p-1>0, \\
f_{19,4}(p) & =380 p^{19}+45 p^{17}+6 p^{12}+p^{7}+p-1<0 .
\end{aligned}
$$

We thus find that $m_{4}=18$.

We now see that the player's strategy is to play a mixed strategy given by Theorem 1 until either the oracle lies or play reaches game $G_{18,4}$. If play reaches game $G_{18,4}$ then the player should bet on heads every time until the coin lands tails or there are no flips remaining. However, if the oracle lies before reaching game $G_{18,4}$ and the player disagrees with the oracle's prediction, then the player will continue to play a mixed strategy given by Theorem 1 until either the oracle lies again or play reaches game $G_{12,3}$, and so on through games $G_{7,2}$ and $G_{2,1}$. 
Because the player and oracle begin with game $G_{20,4}$, the player would like to find the expected value of this game. This involves evaluating $g_{20,4}(x)$ at $x=p$. We first compute $g_{n, 4}(x)$ :

$$
\begin{aligned}
g_{n, 4}(x)= & \sum_{i_{1}=1}^{n-18} \sum_{i_{2}=1}^{n-i_{1}-12} \sum_{i_{3}=1}^{n-i_{1}-i_{2}-7} \sum_{i_{4}=1}^{n-i_{1}-i_{2}-i_{3}-2} x^{n}+\sum_{i_{1}=1}^{n-18} \sum_{i_{2}=1}^{n-i_{1}-12} \sum_{i_{3}=1}^{n-i_{1}-i_{2}-7} x^{n-2} \\
& +\sum_{i_{1}=1}^{n-18} \sum_{i_{2}=1}^{n-i_{1}-12} x^{n-7}+\sum_{i_{1}=1}^{n-18} x^{n-12}+x^{n-18} .
\end{aligned}
$$

When $n=20$, we have $g_{20,4}(p)=870 p^{20}+101 p^{18}+13 p^{13}+2 p^{8}+p^{2}$. Therefore, the expected payoff to the player in the game $G_{20,4}$ when $p=\frac{2}{3}$ is

$$
E_{20,4}=\frac{(2 p)^{20}}{g_{20,4}(p)}=\frac{274877906944}{801309129} \approx 343.036
$$

\section{Open questions}

The question arises, is there a simple relationship between the integers $k$ and $m_{k}$ ? In the extended example above, we saw that $m_{1}=2, m_{2}=7, m_{3}=12$, and $m_{4}=18$. Further calculations show that $m_{5}=24, m_{6}=31, m_{7}=37, m_{8}=43$, and so on. Indeed, for larger values of $k$, the value of $m_{k}-m_{k-1}$ is almost always 7 (and occasionally 8).

More precisely, the question is, does

$$
\lim _{k \rightarrow \infty} \frac{m_{k}}{k}
$$

exist for all $p>\frac{1}{2}$ ? Extensive numerical calculations with MATHEMATICA ${ }^{\circledR}$ and MAPLE ${ }^{\circledR}$ suggest that the answer is 'yes'. Indeed, when $p=\frac{2}{3}$, it appears that $\lim _{k \rightarrow \infty}\left(m_{k} / k\right) \approx 7.1$. This suggests that, when $p=\frac{2}{3}$ and $n$ is large, to maintain credibility, the oracle needs to lie an average of at least once in every 7.1 coin tosses. (This may sound contrary to everyday experience, but, by lying, the oracle is depleting a valuable resource: its ability to lie.)

Table 1 summarizes some numerical results obtained from MAPLE, using values of $k$ up to 10000 .

TABLe 1.

\begin{tabular}{cc}
\hline$p$ & $\lim _{k \rightarrow \infty}\left(m_{k} / k\right)$ \\
\hline 0.55 & $\approx 3.5$ \\
0.60 & $\approx 4.8$ \\
0.65 & $\approx 6.5$ \\
0.70 & $\approx 8.7$ \\
0.75 & $\approx 11.9$ \\
0.80 & $\approx 17.0$ \\
0.85 & $\approx 26.0$ \\
0.90 & $\approx 45.4$ \\
0.95 & $\approx 110.3$ \\
0.99 & $\approx 731.0$ \\
\hline
\end{tabular}


Lastly, a curious phenomenon occurs in the neighborhood of $p=\frac{1}{2}$. When $p=\frac{1}{2}, m_{k}=k$ for all $k \geq 1$ and, therefore, $m_{k}-m_{k-1}=1$ for all $k \geq 1$. However, if $p>\frac{1}{2}$ then numerical calculations indicate that $\lim _{k \rightarrow \infty}\left(m_{k} / k\right) \geq 2$. This tells us that if the coin is biased, even ever so slightly, then the oracle must lie at least half the time in order to maintain credibility.

\section{Acknowledgement}

The authors thank an anonymous referee for several useful suggestions.

\section{References}

[1] Gossner, O. And Vieille, N. (2002). How to play with a biased coin? Games Econom. Behavior 41, $206-226$.

[2] Koether, R. (2007). The generalized paper-scissors-stone game. Preprint.

[3] Koether, R. T. and Osoinach, J. K. (2005). Outwitting the lying oracle. Math. Mag. 78, 98-109.

[4] Pendergrass, M. (2009). A path guessing game with wagering. Preprint. Available at http://arxiv.org/ abs/0907.2196.

[5] Ravikumar, B. (2005). Some connections between the lying oracle problem and Ulam's search problem. In Proc. Australasian Workshop Combinatorial Algorithms (Ballarat, September 2005), eds J. Ryan et al., University of Ballarat, pp. 269-277.

[6] Rivest, R. L., Mayer, A. R., Kleitman, D. J. and Winklemann, K. (1980). Coping with errors in binary search procedures. J. Comput. System Sci. 20, 396-404. 